\title{
Aortic valve repair: Intraoperative evaluation of valve geometry by angioscopy
}

\author{
Konstantinos Tsagakis, MD, ${ }^{\mathrm{a}}$ Jaroslav Benedik, MD, ${ }^{\mathrm{a}}$ Gebrine El Khoury, $\mathrm{MD}, \mathrm{PhD},{ }^{\mathrm{b}}$ and \\ Heinz Jakob, MD, PhD, ${ }^{a}$ Essen, Germany, and Brussels, Belgium
}

See related commentary on pages $1668-9$.

Aortic valve (AV) repair still represents a surgical challenge. To obtain optimal AV function, adequate interaction of all anatomic components is crucial. This includes the integrity and geometry of the AV leaflets as well as the dimension of the aortic annulus, sinotubular junction, and ventriculoaortic junction. The result of the repair has to be done directly during cardioplegic arrest with a depressurized aortic root and left ventricle. Experience is required to predict the result of the repair and to avoid secondary aortic clamping for valve re-repair or replacement. ${ }^{1-3}$ Especially in patients requiring additional surgical procedures, like in acute type-A aortic dissection with concomitant AV regurgitation, a secondary cardioplegic arrest time would increase the overall risk. We report a novel technique using a videoscope for intraoperative evaluation of the AV geometry under diastolic conditions during cardioplegic arrest after replacement of the ascending aorta.

\section{SURGICAL TECHNIQUE}

A flexible autoclavable videoscope (length, $60 \mathrm{~cm}$; diameter, $5 \mathrm{~mm}$ ) (BF type Q180-AC; Olympus, Tokyo, Japan) called an angioscope was used. ${ }^{4}$ Following AV repair and replacement of the ascending aorta the angioscope was placed within the lumen of the vascular graft parallel to a cannula for administration of about $500 \mathrm{~mL}$ cold crystalloid cardioplegia (Custodiol; Dr Franz Köhler Chemie GmbH, Alsbach-Hähnlein, Germany). The application of the translucent solution was started and the vascular graft was clamped beside the angioscope and the cannula. The solution was administered using a pressure cuff and the left ventricle was simultaneously vented. Pressure in the aortic root of at

From the Department of Thoracic and Cardiovascular Surgery, ${ }^{a}$ West-German Heart Center Essen, University Hospital Essen, Essen, Germany; and Department of Cardiovascular and Thoracic Surgery, ${ }^{\text {b }}$ Cliniques Universitaires Saint-Luc, Brussels, Belgium.

Disclosures: Authors have nothing to disclose with regard to commercial support.

Received for publication Nov 25, 2014; revisions received Jan 8, 2015; accepted for publication Jan 29, 2015; available ahead of print March 7, 2015.

Address for reprints: Konstantinos Tsagakis, MD, Department of Thoracic and Cardiovascular Surgery, West-German Heart Center Essen, University Hospital Essen, Hufelandstr 55, 45147 Essen, Germany (E-mail: konstantinos.tsagakis@ uk-essen.de).

J Thorac Cardiovasc Surg 2015;149:1666-8

$0022-5223 / \$ 36.00$

Copyright (c) 2015 by The American Association for Thoracic Surgery

http://dx.doi.org/10.1016/j.jtcvs.2015.01.059 least $60 \mathrm{~mm} \mathrm{Hg}$ was established, depending on the leakage beside the clamp and into the coronary arteries. Washout of remaining blood into the coronary arteries leads to clear visualization of the AV in the diastolic position. Therefore, the symmetry of the leaflets' coaptation was evaluated under physiologic conditions. Asymmetric coaptation due to prolapse as well as a leakage could be identified in $<2$ minutes.

\section{CLINICAL EXPERIENCE}

The AV angioscope was used from February 2014 to September 2014 in 26 patients with AV regurgitation combined with acute type-A aortic dissection or ascending aortic aneurysm. The regurgitation was quantified preand postoperatively by transesophageal echocardiography (TEE). Perfect visualization and examination of the valve geometry by the angioscope was successful in all patients (Figure 1). In patients with symmetric leaflet coaptation intraoperatively, no residual regurgitation was observed by TEE postoperatively. Minimal central leakage with symmetric leaflet coaptation resulted in a mild regurgitation in TEE. Asymmetric leaflet coaptation without or with leakage demonstrated mild or moderate residual regurgitation, respectively (Table 1). Two patients with moderate and 1 with severe regurgitation underwent secondary clamping (for valve re-repair in 1 and replacement in 2).

\section{COMMENT}

AV testing after repair is usually performed by a flush maneuver using a syringe of saline. This simple method is efficient in very experienced hands to predict the geometry of the valve or to add further repair techniques. However, this technique fails to simulate the leaflets' coaptation under diastolic pressure. The angioscopic valve evaluation enables the visualization of the repair, the position of the free-edge leaflets level in diastole, and the identification of residual leakage. In case of prolapse, additional central free-edge plication can be performed and controlled immediately. Severe defects of the valve justifying replacement can be recognized. The procedure is not time consuming and can be performed with all kinds of thoracic videoscopes, flexible or stiff. The quality of the visualization is satisfactory but care has to be taken to keep a distance between the tip of the videoscope and the valve to have an appropriate field of view. An extensive shortening of the vascular graft up to the sinutubular junction in AV reimplantation technique can result in a limited field of view. The identification of the coronary orifices enables the orientation in the 


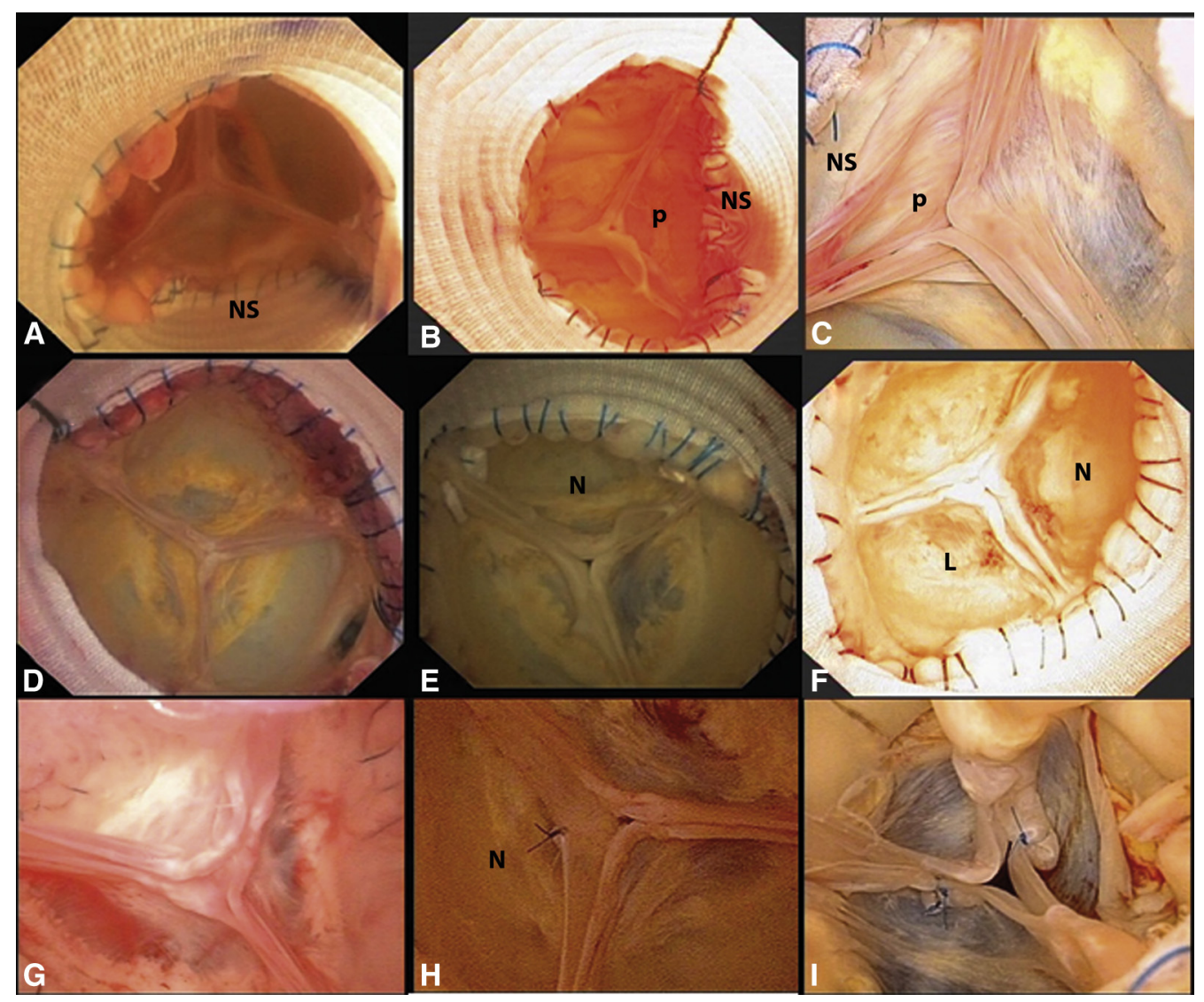

FIGURE 1. Angioscopic view of the aortic valve (AV) in the diastolic position after repair. A-C, AV findings in patients with acute type-A aortic dissection after valve remodeling by noncoronary sinus $(N S)$ and supracoronary aortic replacement. A, Symmetric leaflet coaptation without leakage resulted in no regurgitation. B, Asymmetric leaflet coaptation due to prolapse $(p)$ of noncoronary leaflet with minimal central leakage resulted in mild regurgitation. $\mathrm{C}, \mathrm{p}$ of noncoronary leaflet resulted in mild regurgitation. D-F, AV findings in acute type-A aortic dissection and aneurysm patients after supracoronary ascending replacement. D, Symmetric leaflet coaptation without leakage resulted in no regurgitation. E, Symmetric leaflet coaptation after resuspension of noncoronary leaflet $(N)$ with central leakage resulted in mild regurgitation. F, Decalcification of left $(L)$ and $\mathrm{N}$ leaflet resulted in symmetric coaptation and no regurgitation. G-I, AV findings after reimplantation of the valve. G, Symmetric leaflet coaptation without leakage resulted in no regurgitation. H, Asymmetric leaflet coaptation with central leakage after $\mathrm{N}$ leaflet plication resulted in mild regurgitation. I, Asymmetric leaflet coaptation and central leakage after plication resulted in severe regurgitation and replacement of the valve.

optical field and the differentiation of the leaflets. Thus, we favor the use of a flexible videoscope. AV angioscopy has become part of our routine as an additional intraoperative diagnostic tool for immediate decision making for ad hoc reintervention before TEE, especially in case of asymmetric leaflet coaptation and residual leakage. In addition, the excellent visualization of the $\mathrm{AV}$ in the diastolic position gives surgeons the option to control the valve geometry after repair and may support the learning curve of $\mathrm{AV}$ repair techniques.

\section{References}

1. David TE, Feindel CM, Bos J. Repair of the aortic valve in patients with aortic insufficiency and aortic root aneurysm. J Thorac Cardiovasc Surg. 1995;109:345-51.

2. de Kerchove L, Glineur D, Poncelet A, Boodhwani M, Rubay J, Dhoore W, et al Repair of aortic leaflet prolapse: a ten-year experience. Eur J Cardiothorac Surg. 2008;34:785-91.

TABLE 1. Correlation between intraoperative angioscopic and postoperative echocardiographic findings

\begin{tabular}{lcccc}
\hline & & \multicolumn{2}{c}{ Postoperative regurgitation based on echocardiography } \\
\cline { 2 - 5 } \multicolumn{1}{c}{ Intraoperative angioscopic findings } & No. of patients & $\mathbf{n}$ & Mild & Moderate/severe \\
\hline Symmetric leaflet coaptation without leakage & 12 & 12 & 0 & 0 \\
Symmetric leaflet coaptation with minimal leakage & 4 & 0 & 4 & 0 \\
Asymmetric leaflet coaptation without leakage & 6 & 1 & 5 & 0 \\
Asymmetric leaflet coaptation with leakage & 4 & 0 & 1 & 3 \\
\hline
\end{tabular}


3. Aicher D, Langer F, Adam O, Tscholl D, Lausberg H, Schäfers HJ. Cusp repair in aortic valve reconstruction: does the technique affect stability? J Thorac Cardiovasc Surg. 2007;134:1533-8.
4. Tsagakis K, Kamler M, Benedik J, Jakob H. Angioscopy—a valuable tool in guiding hybrid stent grafting and decision making during type A aortic dissection surgery. Eur J Cardiothorac Surg. 2010;38:507-9.

\title{
EDITORIAL COMMENTARY
}

\section{Better seeing is more believing: Angioscopy in aortic valve repair}

\author{
Hans-Hinrich Sievers, MD
}

See related article on pages $1666-8$.

In this issue of the Journal, Tsagakis and colleagues ${ }^{1}$ very nicely present their first experience with intraoperative evaluation of valve geometry by angioscopy during aortic valve repair. Reviewing this article, at first I enjoyed their angioscopic pictures; later, however, I became a little skeptical because (1) the decision for rerepair was not based on the angioscopic findings but on conventional postoperative echocardiography, (2) the angioscopically observed geometric abnormalities were not validated by instantaneous echocardiography, (3) the pictures were 2-dimensional and did not allow assessment of leaflet

From the Department of Cardiac and Thoracic Vascular Surgery, University of Lübeck, Lübeck, Germany.

Disclosures: Author has nothing to disclose with regard to commercial support.

Received for publication March 20, 2015; accepted for publication March 20, 2015; available ahead of print April 21, 2015.

Address for reprints: Hans-Hinrich Sievers, MD, Department of Cardiac and Thoracic Vascular Surgery, University of Lübeck, Ratzeburger Allee 160, 23538 Lübeck, Germany (E-mail: Hans-Hinrich.Sievers@uksh.de).

J Thorac Cardiovasc Surg 2015;149:1668-9

$0022-5223 / \$ 36.00$

Copyright (c) 2015 by The American Association for Thoracic Surgery http://dx.doi.org/10.1016/j.jtcvs.2015.03.037 height, and (4) the level of the diastolic pressure was not clear.

Repair of the aortic valve is attractive, but it is still a developing and difficult technique, at least for me, even after more than 600 aortic valve repairs. Why? The anatomy and dynamics of the aortic root are extremely complex, resulting in a highly sophisticated, fine-tuned concert of all components, mainly the aortoventricular and sinotubular junction, the interleaflet triangles, and the leaflets, ${ }^{2}$ within relatively small dimensional boundaries and tolerances. In diastole, the leaflets coapt perfectly in absolute parallel, without any folds or irregularities (Figure 1, A). This morphologic unit between the heart and the ascending aorta is the optimal solution for a long-lasting stenosis- and regurgitation-free valve mechanism, not producible by human technology. All dimensional abnormalities will lead to more or less valve dysfunction and in the worst case to insufficiency, whereby one or multiple anatomic levels or components can be affected, each needing consideration during repair. Furthermore, the quality, the pliability, the age-related increase in stiffness, ${ }^{3}$ the height, or other pathologies of the leaflets influence the results of repair. Consequently, the complexity of repair has different degrees, all aimed at creating a perfect coaptation line and area of 2 to $3 \mathrm{~mm}$ for complete sealing without stenosis. Even little folds in the free edge of a single leaflet can cause regurgitation, especially if the tissue is
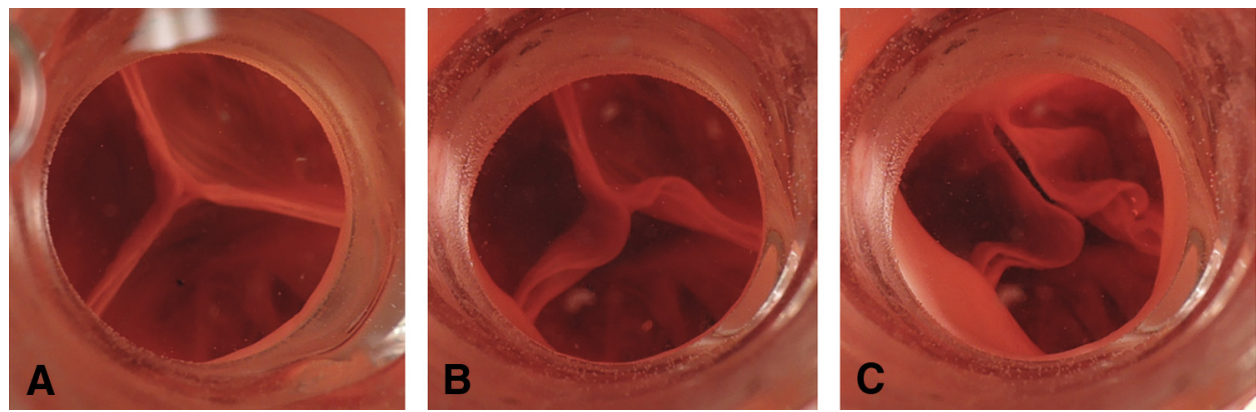

FIGURE 1. Endoscopic view of the normal aortic root at different diastolic pressures. A, At $80 \mathrm{~mm} \mathrm{Hg}$; B, at $40 \mathrm{~mm} \mathrm{Hg}$; C, at $0 \mathrm{~mm} \mathrm{Hg}$. Note the straight parallelism of the coaptation area of the leaflets in panel A, which becomes irregular when the diastolic pressure is reduced, making the assessment of the valve difficult. 